\title{
Correlação entre fechamento velofaríngeo e dimensões nasofaríngeas após cirurgia de retalho faríngeo avaliados por meio da técnica fluxo-pressão
}

\author{
Correlation between velopharyngeal closure and \\ nasopharyngeal dimensions after pharyngeal flap surgery \\ assessed by pressure-flow technique
}

Flávia Fernandes Lanziani ${ }^{1}$, Renata Paciello Yamashita², Ana Paula Fukushiro ${ }^{3}$, Inge Elly Kiemle Trindade ${ }^{4}$

\begin{abstract}
RESUMO
Objetivo: Verificar se a área dos orifícios velofaríngeos obtida após o retalho faríngeo (RF) durante a respiração de repouso (AVFr) é um indicador do grau de fechamento velofaríngeo durante a fala (AVFf). Métodos: Os sujeitos foram 62 pacientes com fissura de palato, associada ou não à fissura de lábio, de ambos os gêneros, com idades entre seis e 32 anos, submetidos ao RF há, pelo menos, 12 meses. AVFr e AVFf foram determinadas por meio da técnica fluxo-pressão. Valores de AVFr inferiores a $0,500 \mathrm{~cm}^{2}$ foram considerados subnormais. A AVFf foi classificada como adequada $\left(0-0,049 \mathrm{~cm}^{2}\right)$, marginal $\left(0,050-0,199 \mathrm{~cm}^{2}\right)$ ou inadequada $(>0,200$ $\mathrm{cm}^{2}$ ). A associação entre os valores de AVFr e AVFf pós-cirúrgicos foi analisada por meio de um modelo de regressão logística. Resultados: Após o RF, 92\% dos pacientes com valores de AVFr subnormais $\left(<0,500 \mathrm{~cm}^{2}\right)$ apresentaram fechamento velofaríngeo adequado. A proporção de pacientes com valores de AVFr normais $\left(>0,500 \mathrm{~cm}^{2}\right)$ e fechamento velofaríngeo adequado foi menor, porém ainda expressiva (55\%). Não houve associação estatisticamente significante entre as duas variáveis. Conclusão: A maioria dos pacientes com retalho largo apresentou fechamento velofaríngeo adequado durante a fala. Entretanto, os resultados mostraram que as dimensões dos orifícios velofaríngeos durante a respiração de repouso não podem predizer a eficácia do retalho faríngeo para a fala.
\end{abstract}

Descritores: Esfíncter velofaríngeo; Nasofaringe; Retalhos cirúrgicos; Período pós-operatório; Insuficiência velofaríngea/cirurgia; Fissura palatina/cirurgia; Rinomanometria; Respiração; Fala

\section{INTRODUÇÃO}

A insuficiência velofaríngea (IVF) é caracterizada por

Trabalho realizado no Laboratório de Fisiologia do Hospital de Reabilitação de Anomalias Craniofaciais da Universidade de São Paulo - HRAC-USP Bauru (SP), Brasil.

(1) Especializanda em Motricidade Orofacial: enfoque em fissura labiopalatina do Hospital de Reabilitação de Anomalias Craniofaciais da Universidade de São Paulo - HRAC-USP - Bauru (SP), Brasil.

(2) Doutora, Fonoaudióloga do Laboratório de Fisiologia e do Setor de Fonoaudiologia do Hospital de Reabilitação de Anomalias Craniofaciais da Universidade de São Paulo - HRAC-USP - Bauru (SP), Brasil.

(3) Doutora, Professora do Departamento de Fonoaudiologia da Faculdade de Odontologia de Bauru da Universidade de São Paulo - USP - Bauru (SP), Brasil; Fonoaudióloga do Laboratório de Fisiologia do Hospital de Reabilitação de Anomalias Craniofaciais da Universidade de São Paulo - HRAC-USP - Bauru (SP), Brasil.

(4) Professora Titular do Departamento de Ciências Biológicas da Faculdade de Odontologia de Bauru e do Hospital de Reabilitação de Anomalias Craniofaciais da Universidade de São Paulo - HRAC-USP - Bauru (SP), Brasil. Endereço para correspondência: Renata Paciello Yamashita. R. Sílvio Marchione, 3-20, Vila Universitária, Bauru (SP), Brasil, CEP: 17012-900.

E-mail: rezeyama@usp.br

Recebido em: 11/5/2009; Aceito em: 3/9/2009 uma falha de origem estrutural na separação entre a cavidade oral e nasal que impossibilita o fechamento velofaríngeo adequado. Como consequência, durante a fala, parte da corrente aérea expiratória sonorizada é desviada para a cavidade nasal, provocando hipernasalidade, emissão de ar nasal e distúrbios articulatórios compensatórios ${ }^{(1-3)}$.

Para propiciar condições anatômicas e favorecer o fechamento velofaríngeo, é realizada a cirurgia primária de palato; porém esta pode não eliminar os sintomas da insuficiência velofaríngea, fazendo-se necessário uma cirurgia secundária do palato que tem como objetivo estreitar o orifício velofaríngeo a fim de permitir um completo fechamento velofaríngeo ${ }^{(4-7)}$.

Existem várias técnicas cirúrgicas empregadas para a correção da IVF, sendo o retalho faríngeo de pedículo superior a mais utilizada. A técnica consiste na construção de uma ponte de tecido miomucoso entre a parede posterior da faringe e o palato mole, delimitando dois orifícios laterais. A base do retalho faríngeo deve ficar no nível do corpo da primeira vértebra cervical, que corresponde à área de transição entre a mucosa faríngea e o início do tecido adenoideano. Está demonstrado através de estudos com nasoendoscopia e videofluoroscopia 
que este é o local de melhor movimento das paredes laterais e posterior da faringe, o que torna o retalho mais eficiente quando está localizado nesta região. A largura do retalho é determinada conforme a intensidade dos movimentos das paredes laterais que devem ser avaliadas antes da cirurgia ${ }^{(7)}$. O objetivo da cirurgia é criar uma obstrução mecânica capaz de separar a cavidade nasal do restante do trato vocal, o que evita a passagem da corrente aérea sonorizada para a cavidade nasal durante a produção dos sons orais. Do ponto de vista acústico, há diminuição da hipernasalidade e, na perspectiva aerodinâmica, obtem-se aumento da pressão intra-oral durante a fala ${ }^{(8)}$. Os dois orifícios posicionados lateralmente ao retalho devem ter diâmetro e permeabilidade suficientes para permitir a respiração nasal em repouso ${ }^{(9)}$.

Muitos estudos relataram a efetividade do retalho faríngeo na redução dos sintomas da fala ${ }^{(4,6,10-18)}$. Por outro lado, o retalho faríngeo está associado a um potencial e significante de comprometimento das vias aéreas superiores, incluindo-se entre esses comprometimentos desde a hiponasalidade, obstrução nasal crônica, até ronco e apnéia obstrutiva do sono, que podem trazer consequências graves aos pacientes ${ }^{(4,10,19-21)}$.

$\mathrm{O}$ efeito do retalho faríngeo sobre a fala e a respiração dos pacientes tem sido estudado há anos, no Laboratório de Fisiologia do Hospital de Reabilitação de Anomalias Craniofaciais (HRAC-USP). Num dos primeiros estudos ${ }^{(4)}$, os autores constataram por meio de avaliação perceptiva e nasométrica, redução da hipernasalidade e da nasalância em $75 \%$ e $60 \%$ dos pacientes submetidos ao retalho faríngeo, respectivamente. Entretanto, 35\% dos pacientes passaram a apresentar hiponasalidade ao exame perceptivo e nasométrico, um sintoma de fala que pode refletir a obstrução nasal. Em outro estudo conduzido no Laboratório ${ }^{(6)}$, empregou-se a avaliação perceptiva da fala, a nasometria e, também, a técnica fluxo-pressão para avaliar os resultados do retalho faríngeo. A autora verificou redução da hipernasalidade em $75 \%$, redução da nasalância em $68 \%$ e diminuição da área velofaríngea associada à melhora do grau de fechamento velofaríngeo, em $66 \%$ dos pacientes. Outros dois estudos, verificaram sinais e sintomas respiratórios indicativos de obstrução respiratória em $53 \%$ adultos de meia-idade após a cirurgia de retalho faríngeo ${ }^{(22)}$, evidências de desordens respiratórias decorrentes da cirurgia em $36 \%$ e redução das dimensões nasofaríngeas para níveis subnormais, em parcela considerável de pacientes adultos jovens ${ }^{(21)}$.

Assim, visto que a técnica do retalho faríngeo apresenta um alto índice de sucesso na eliminação dos sintomas de fala da IVF, mas que, por outro lado, é a técnica mais associada a complicações respiratórias, o presente estudo pretendeu verificar, por meio da técnica fluxo-pressão, se as dimensões dos orifícios velofaríngeos durante a respiração de repouso podem ser consideradas indicadores do grau de fechamento velofaríngeo durante a fala.

\section{MÉTODOS}

Este estudo foi conduzido no Laboratório de Fisiologia do HRAC-USP e recebeu aprovação do Comitê de Ética em Pesquisa em Seres Humanos da instituição em 28/05/2008ofício no 162/2008 - SVAPEPE-CEP.

\section{Casuística}

Trata-se de estudo retrospectivo no qual foram analisadas as avaliações aerodinâmicas (técnica fluxo-pressão) de 62 pacientes com fissura de palato, associada ou não à fissura de lábio, de ambos os sexos, com idade entre cinco e 32 anos (17 anos, em média), submetidos à cirurgia de retalho faríngeo de pedículo superior para correção da IVF há pelo menos um ano.

\section{Procedimentos}

As áreas dos orifícios velofaríngeos obtidas durante a respiração de repouso (AVFr) e durante a fala (AVFf) foram determinadas por meio da técnica fluxo-pressão, conhecida como rinomanometria anterior modificada, utilizando um sistema computadorizado PERCI-SARS (Microtronics Corp, versão 3.30). A técnica baseia-se no fato de que a área de secção transversa mínima de uma constrição (ou orifício) pode ser estimada pela medida simultânea da pressão diferencial entre os dois lados da constrição e, do fluxo aéreo que a atravessa. A Figura 1 mostra a representação esquemática da instrumentação utilizada para estimar as dimensões dos orifícios velofaríngeos.

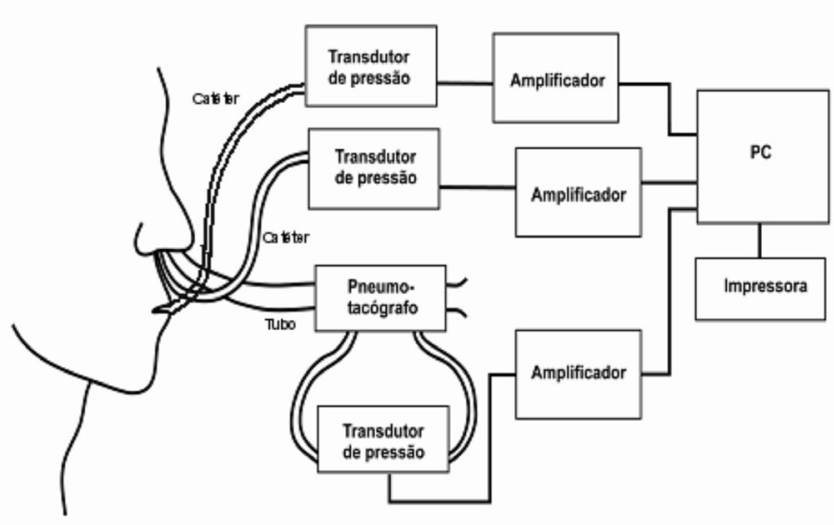

Fonte: Trindade IEK, Yamashita, RP, Gonçalves CGAB. Diagnóstico instrumental da disfunção velofaríngea. In: Trindade IEK, Silva Filho OG, coordenadores. Fissura Labiopalatinas: uma abordagem interdisciplinar. São Paulo: Editora Santos; 2007. p. 123-63.

Figura 1. Instrumentação para determinação das áreas dos orifícios velofaríngeos durante a respiração e a fala (Sistema PERCI-SARS®, Microtronics Corp., Chapel Hill. NC, USA)

\section{Área dos orifícios velofaríngeos durante a respiração de repouso (AVFr)}

O exame é realizado durante a respiração nasal. A pressão diferencial através da região nasofaríngea é medida posicionando-se um cateter no interior da boca e outro na narina de menor fluxo. O cateter nasal é mantido em posição por um obturador nasal que bloqueia a narina, criando uma coluna de ar estática, permitindo assim, a medida da pressão da nasofaringe. O fluxo aéreo nasal é medido por meio de um tubo plástico posicionado na narina de maior fluxo. O tubo é conectado ao pneumotacógrafo aquecido e ligado a um terceiro transdutor cujos sinais são enviados ao sistema para análise por programa computadorizado específico. As medidas são 
Tabela 1. Valores da área dos orifícios velofaríngeos durante a respiração de repouso de acordo com o grau de fechamento velofaríngeo obtidos após a cirurgia de retalho farínge

\begin{tabular}{llllccc}
\hline Fechamento velofaríngeo & $\mathrm{N}$ & Média & $\mathrm{DP}$ & Mínimo & Mediana & Máximo \\
\hline Adequado & 39 & 0,601 & 0,212 & 0,178 & 0,593 & 0,960 \\
Marginal & 11 & 0,651 & 0,090 & 0,548 & 0,652 & 0,800 \\
Inadequado & 12 & 0,722 & 0,122 & 0,448 & 0,793 & 0,804 \\
\hline Total & 62 & 0,633 & 0,185 & 0,178 & 0,635 & 0,960 \\
\hline
\end{tabular}

Modelo de regressão logística - $p<0,05$. Valores expressos em $\mathrm{cm}^{2}$.

Legenda: DP = desvio-padrão.

feitas nos picos dos fluxos inspiratório e expiratório em duas a quatro respirações sucessivas. A área considerada para análise corresponde à média dessas múltiplas medidas e é calculada pela equação: $\mathrm{A}=\mathrm{V} / \mathrm{k}(2 \Delta \mathrm{P} / \mathrm{d})^{1 / 2}$, onde $\mathrm{A}=$ área dos orifícios em $\mathrm{cm}^{2} ; \mathrm{V}=$ fluxo nasal em $\mathrm{cm}^{3} / \mathrm{s} ; \mathrm{K}=0,65 ; \Delta \mathrm{P}=$ pressão oral-nasal em dinas $/ \mathrm{cm}^{2} ; \mathrm{d}=$ densidade do ar $\left(0,001 \mathrm{~g} / \mathrm{cm}^{3}\right)$. Para fins de análise, foi adotado como referência o valor de $0,500 \mathrm{~cm}^{2(21)}$. Assim, os valores de AVFr abaixo de $0,500 \mathrm{~cm}^{2}$ foram considerados subnormais.

\section{Área dos orifícios velofaríngeos durante a fala (AVFf)}

A área é determinada durante a produção da consoante plosiva "p" inserida no vocábulo "rampa". São avaliadas de quatro a seis emissões sucessivas de cada produção por indivíduo. A área considerada para análise representa a média dessas produções múltiplas e é calculada pelo próprio programa utilizando a mesma equação citada anteriormente.

Para fins de análise, os valores de AVFf foram classificados de acordo com o critério adaptado de Warren ${ }^{(23)}: 0$ a $0,049 \mathrm{~cm}^{2}$ $=$ fechamento velofaríngeo adequado; 0,050 a $0,199 \mathrm{~cm}^{2}=$ fechamento velofaríngeo marginal e, $\geq 0,200 \mathrm{~cm}^{2}=$ fechamento velofaríngeo inadequado.

\section{Análise dos dados}

As áreas dos orifícios velofaríngeos foram expressas em $\mathrm{cm}^{2}$. A correlação entre os valores de AVFr e AVFf foi analisada por meio de um modelo de regressão logística ${ }^{(24)}$. O nível de significância adotado foi menor que 0,05 .

\section{RESULTADOS}

Após a cirurgia de retalho faríngeo, verificou-se que a AVFr média $( \pm \mathrm{DP})$, obtida nos 62 pacientes analisados foi de $0,633 \pm 0,185 \mathrm{~cm}^{2}$, dos quais $49(79 \%)$ apresentaram valores normais $\left(>0,500 \mathrm{~cm}^{2}\right)$ e $13(21 \%)$ apresentaram valores de AVFr considerados subnormais $\left(<0,500 \mathrm{~cm}^{2}\right)$. Com relação ao efeito do retalho faríngeo sobre a fala, a AVFf média $( \pm D P)$ foi de $0,144 \pm 0,284 \mathrm{~cm}^{2}$. Analisando-se individualmente o grau de fechamento velofaríngeo obtido após a cirurgia verificou-se que $39(63 \%)$ pacientes passaram a apresentar fechamento velofaríngeo adequado, 11 (18\%) apresentaram fechamento velofaríngeo marginal, e 12 (19\%), fechamento inadequado. A AVFr média $( \pm \mathrm{DP})$ para cada uma das categorias de fechamento velofaríngeo está apresentada na Tabela 1 , juntamente com os valores medianos, mínimos e máximos. Verificou-se AVFr de $0,601 \pm 0,212 \mathrm{~cm}^{2}$ para o grupo com fechamento adequado,
$0,651 \pm 0,090 \mathrm{~cm}^{2}$ para o grupo marginal e $0,722 \pm 0,122 \mathrm{~cm}^{2}$ para o grupo com fechamento velofaríngeo inadequado. Os boxplots da AVFr segundo o grau de fechamento velofaríngeo, apresentados na Figura 2, ilustram estes resultados.

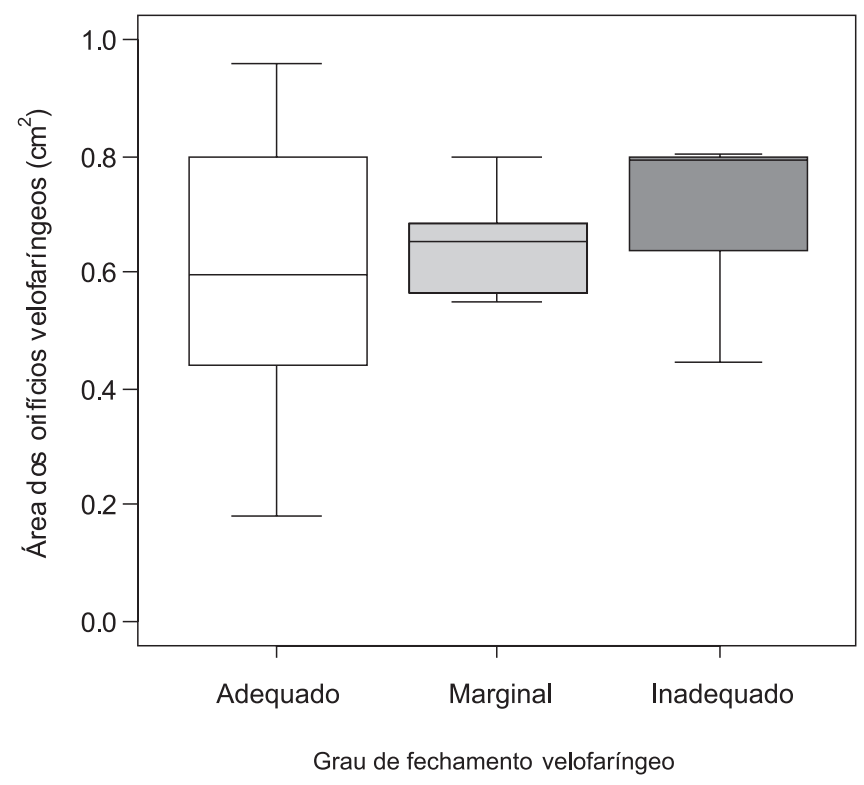

Figura 2. Boxplots referentes aos valores de área dos orifícios velofaríngeos durante a respiração, distribuídos de acordo com o grau de fechamento velofaríngeo (adequado, marginal e inadequado) obtidos após a cirurgia de retalho faríngeo

No grupo de pacientes com valores subnormais à respiração, verificou-se que, $92 \%$ (12/13) apresentaram fechamento velofaríngeo adequado e $8 \%$ (1/13) mantiveram-se com fechamento inadequado após a cirurgia. Já no grupo de pacientes com AVFr normal, 55\% (27/49) apresentaram fechamento velofaríngeo adequado, $22 \%$ (11/49) com fechamento marginal e 22\% (11/49) com fechamento inadequado. Ao investigar a correlação entre as dimensões nasofaríngeas na respiração e na fala, os resultados do modelo de regressão logística mostraram não haver associação estatisticamente significante entre as duas variáveis.

\section{DISCUSSÃO}

Considerando-se que o retalho faríngeo é o procedimento cirúrgico mais utilizado na correção da insuficiência velofaríngea residual, especialmente nos casos mais severos e que tal cirurgia, ao mesmo tempo, está frequentemente associada ao comprometimento das vias aéreas superiores, é de funda- 
mental importância que cirurgiões e clínicos estejam atentos para tal risco no momento da escolha deste procedimento. A técnica de retalho faríngeo tem por objetivo criar dois orifícios laterais, os quais, idealmente, devem permanecer abertos durante a respiração nasal e a produção de consoantes nasais, e manterem-se fechados durante a produção dos sons orais da fala. É sabido que o sucesso da cirurgia de retalho faríngeo aumenta significativamente quando o retalho é construído "sob medida", ou seja, conhecendo-se o local de maior movimento das paredes laterais da faringe e o tamanho do gap velofaríngeo ${ }^{(25)}$. Assim, a nasoendoscopia é o método instrumental eleito para avaliar as condições pré-cirúrgicas, uma vez que permite definir, ainda que subjetivamente, a largura e posição do retalho, determinados a partir da intensidade de movimento das paredes laterais ${ }^{(26)}$.

Entretanto, apesar de ser possível modelar o tamanho dos orifícios na cirurgia, tanto o retalho quanto os orifícios laterais podem sofrer modificações após a cirurgia devido à cicatrização e migração pós-operatória das estruturas ${ }^{(9)}$. Uma vez que o processo de cicatrização e contração dos tecidos não pode ser controlado e mudanças no tamanho e forma dos orifícios e do retalho são imprevisíveis após a cirurgia, tais fatores podem, em alguns casos, levar à falha da cirurgia $^{(27)}$, resultando em sintomas respiratórios e de fala variáveis. Quando o retalho fica muito estreito, a hipernasalidade e os demais sintomas da IVF podem persistir em função da impossibilidade de total fechamento dos orifícios. Por outro lado, o insucesso do retalho faríngeo pode ser decorrente da situação inversa, na qual os orifícios laterais mantem-se estreitados prejudicando, assim, a respiração nasal de repouso. Nesses casos, os sintomas mais comuns são a respiração oral, o ronco durante o sono, a apnéia obstrutiva do sono e, ainda, a hiponasalidade ${ }^{(9,21)}$. Como consequência das mudanças na anatomia e fisiologia do esfíncter velofaríngeo, estes sintomas podem aparecer imediatamente após a cirurgia, agravados pelo edema pós-cirúrgico e persistirem ao longo do tempo, sendo necessária, em alguns casos, a revisão da cirurgia priorizando-se a respiração em detrimento da fala. Os procedimentos mais utilizados na tentativa de solucionar a obstrução são aqueles que promovem o aumento dos orifícios laterais do retalho ou, em casos mais severos, a ressecção total do pedículo do retalho. Em estudo realizado para investigar as causas da indicação da revisão cirúrgica do retalho faríngeo no HRAC-USP(5) verificou-se que as queixas respiratórias ocorridas, provavelmente, pela obstrução das vias aéreas representaram $62 \%$ das 93 revisões cirúrgicas, sendo a principal causa da indicação cirúrgica, seguida pela persistência dos sintomas de IVF (34\%) e a combinação de ambos os fatores (4\%).

Há muitos anos tem-se investigado os efeitos da cirurgia sobre a fala e a respiração utilizando julgamento perceptivo associado ou não a métodos objetivos ${ }^{(10-17,28,29)}$. As repercussões do retalho faríngeo têm sido objeto de estudo, também, no Laboratório de Fisiologia do HRAC-USP ${ }^{(4,6,21,22)}$. Estudos utilizando avaliação perceptiva combinada à avaliação nasométrica e aerodinâmica, comprovaram o alto índice de sucesso do retalho faríngeo (75\% dos casos) na redução da hipernasalidade, nos quais a avaliação instrumental confirmou os resultados perceptivos no julgamento da função velofaríngea ${ }^{(4,6)}$.
A técnica fluxo-pressão foi, também, empregada para avaliar o efeito do retalho faríngeo sobre a permeabilidade nasal em pacientes adultos de meia idade ${ }^{(22)}$, e mais recentemente, em indivíduos jovens ${ }^{(21)}$. Em ambas as faixas etárias confirmouse que o retalho faríngeo leva à redução permanente das dimensões nasofaríngeas e, pode ainda, ocasionar obstrução das vias aéreas superiores e queixas respiratórias. Embora, neste último estudo não tenha sido possível definir um valor mínimo "de normalidade" da área dos orifícios velofaríngeos durante a respiração, os autores verificaram que a maioria dos pacientes que relataram queixas respiratórias pós-cirúrgicas apresentou áreas reduzidas, favorecendo a hipótese de uma relação entre essas duas variáveis. Ainda assim, para facilitar a interpretação dos dados, adotou-se como referência, o valor de $0,500 \mathrm{~cm}^{2}$, com base em estudo anterior ${ }^{(30)}$, no qual crianças e adultos sem fissura e sem obstrução respiratória apresentaram um valor mínimo de área nasofaríngea de $0,570 \mathrm{~cm}^{2}$, à avaliação aerodinâmica. Este foi também o critério utilizado no presente estudo.

A técnica fluxo-pressão tem a vantagem de ser um método não invasivo, que não traz riscos ao paciente e permite mensurar as dimensões do espaço nasofaríngeo durante as funções da fala e da respiração por meio da análise dos níveis de fluxos e pressões envolvidos nessas funções. Pelo fato de os processos aerodinâmicos serem responsáveis por parte dos mecanismos de produção da fala, tal método constitui uma importante ferramenta no diagnóstico e acompanhamento do tratamento cirúrgico de pacientes com IVF. No que se refere ao mecanismo velofaríngeo, a técnica permite inferir o potencial anatômico da velofaringe durante a fala, tendo sua aplicação recomendada pela American Cleft Palate Association, na aferição dos resultados cirúrgicos do retalho faríngeo.

Apesar da vasta literatura a respeito dos efeitos do retalho faríngeo sobre a fala e a respiração, o estudo da relação entre as dimensões dos orifícios velofaríngeos e o grau de fechamento velofaríngeo, nunca havia sido abordado.

Considerando-se a relevância do assunto, o presente estudo teve por objetivo investigar, por meio da técnica fluxo-pressão, se é possível predizer o grau de fechamento velofaríngeo aferido durante a fala, a partir das dimensões dos orifícios velofaríngeos obtidos durante a respiração de repouso, após a cirurgia. Numa primeira análise, verificou-se que em $79 \%$ dos pacientes, os orifícios velofaríngeos permaneceram com dimensões suficientes para uma adequada respiração, após a cirurgia. Já a redução das dimensões ocorreu em $21 \%$, proporção menor que os $36 \%$ encontrados em estudo que correlacionou queixas respiratórias às dimensões do espaço nasofaríngeo ${ }^{(21)}$. Neste grupo com valores subnormais na respiração, a maioria (92\%) apresentou fechamento velofaríngeo adequado sugerindo, num primeiro momento, haver relação entre as dimensões dos orifícios velofaríngeos durante a respiração com o grau de fechamento velofaríngeo durante a fala. Por outro lado, a análise individual dos dados mostrou que considerável parcela de pacientes $(55 \%)$ que permaneceram com os orifícios velofaríngeos normais, também apresentou fechamento velofaríngeo adequado, mostrando assim, que as dimensões dos orifícios velofaríngeos no repouso não são suficientes para predizer o grau de fechamento velofaríngeo 
para a fala. O retalho largo parece favorecer o fechamento velofaríngeo adequado, entretanto, não é uma condição obrigatória, ou seja, o fechamento velofaríngeo completo ocorre mesmo na presença de um retalho não obstrutivo. Esta, aliás, é a situação ideal após a cirurgia de retalho faríngeo: adequar a função velofaríngea sem obstruir as vias aéreas superiores.

Sendo assim, a capacidade de previsão da função velofaríngea não está relacionada somente à presença de orifícios velofaríngeos estreitos. Fatores como altura adequada do retalho faríngeo, bom movimento das paredes laterais da faringe e fonoterapia pós-operatória devem ser considerados.

\section{CONCLUSÃO}

Embora a maioria dos pacientes com áreas subnormais durante a respiração de repouso tenha alcançado o fechamento velofaríngeo adequado durante a fala, os resultados do presente estudo mostraram que as dimensões dos orifícios velofaríngeos durante a respiração de repouso não podem predizer a eficácia do retalho faríngeo para a fala.

\begin{abstract}
Purpose: To investigate whether postoperative velopharyngeal orifice area during nasal breathing at rest (VPAb) can predict velopharyngeal closure during speech (VPAs). Methods: The subjects were 62 patients with cleft palate, associated or not with cleft lip, of both genders, with ages between six and 32 years, who underwent pharyngeal flap surgery (PFS) at least 12 months before the evaluation performed for this study. VPAb and VPAs were assessed using the pressure-flow technique. VPAb values below $0.500 \mathrm{~cm}^{2}$ were considered subnormal. VPAs was categorized as adequate $\left(0-0.049 \mathrm{~cm}^{2}\right)$, borderline $\left(0.050-0.199 \mathrm{~cm}^{2}\right)$ or inadequate $\left(\geq 0.200 \mathrm{~cm}^{2}\right)$. A logistic regression model analyzed the association between VPAb and VPAs. Results: After PFS, 92\% of the patients with subnormal VPAb values $\left(<0.500 \mathrm{~cm}^{2}\right)$ presented adequate velopharyngeal closure. The percentage of patients with normal VPAb values $\left(>0.500 \mathrm{~cm}^{2}\right)$ who also presented adequate closure was smaller, but still significant $(55 \%)$. The association between VPAb and VPAs values was not statistically significant. Conclusion: Most patients with large flaps presented adequate velopharyngeal closure during speech. However, the findings show that the velopharyngeal orifice area during breathing is not a good predictor of the effectiveness of the pharyngeal flap for speech.
\end{abstract}

Keywords: Velopharyngeal sphincter; Nasopharynx; Surgical flaps; Postoperative period; Velopharyngeal insufficiency/surgery; Cleft palate/surgery; Rhinomanometry; Respiration; Speech

\title{
REFERÊNCIAS
}

1. Trindade IEK, Trindade Junior AS. Avaliação funcional da inadequação velofaríngea. In: Carreirão S, Lessa S, Zanini AS. Tratamento das fissuras labiopalatinas. 2a ed. Rio de Janeiro: Revinter; 1996. p. 223-35.

2. Kummer AW. Velopharyngeal dysfunction (VPD) and resonance disorders. In: Kummer AW. Cleft palate \& craniofacial anomalies: effects of speech and resonance. San Diego: Singular; 2001. p. 145-76.

3. Genaro KF, Fukushiro AP, Suguimoto MLFCP. Avaliação e tratamento dos distúrbios da fala. In: Trindade IEK, Silva Filho OG. Fissuras labiopalatinas: uma abordagem interdisciplinar. São Paulo: Santos; 2007. p. 109-22.

4. Zuiani TBB, Trindade IEK, Yamashita RP, Trindade Junior AS. The pharyngeal flap surgery in patients with velopharyngeal insufficiency: perceptual and nasometric speech assessment. Braz J Dysmorphol Speech Disord. 1998;2(1):31-42.

5. Battaiola CM, Yamashita RP. Revisão cirúrgica do retalho faríngeo. Salusvita. 2005;24(1):75-96.

6. Fukushiro AP. Análise perceptiva, nasométrica e aerodinâmica da fala de indivíduos submetidos à cirurgia de retalho faríngeo para a correção da insuficiência velofaríngea [tese]. Bauru(SP): Hospital de Reabilitação de Anomalias Craniofaciais, Universidade de São Paulo; 2007.

7. Rocha DL. Tratamento cirúrgico da insuficiência velofaríngea. In: Trindade IEK, Silva Filho OG. Fissuras labiopalatinas: uma abordagem interdisciplinar. São Paulo: Santos; 2007. p. 109-22.

8. Riski JE, Ruff GL, Georgiade GS, Barwich WL, Edwards PD. Evalution of the sphincter pharyngoplasty. Cleft Palate Craniofac J. 1992;29(3):254-61.

9. Witt PD, D'Antonio LL. Velopharyngeal insufficiency and secondary palatal management. A new look at an old problem. Clin Plast Surg. 1993;20(4):707-21
10. Sloan GM. Posterior pharyngeal flap and sphincter pharyngoplasty: the state of the art. Cleft Palate Craniofac J. 2000;37(2):112-22.

11. Seagle MB, Mazaheri MK, Dixon-Wood VL, Williams WN. Evaluation and treatment of velopharyngeal insufficiency: the University of Florida experience. Ann Plast Surg. 2002;48(5):464-70.

12. Tönz M, Schmid I, Graf M, Mischler-Heeb R, Weissen J, Kaiser G. Blinded speech evaluation following pharyngeal flap surgery by speech pathologists and lay people in children with cleft palate. Folia Phoniatr Logop. 2002;54(6):288-95.

13. Ysunza A, Pamplona MC, Mendoza M, Molina F, Martinez P, GarcíaVelasco M, et al. Surgical treatment of submucous cleft palate: a comparative trial of two modalities for palatal closure. Plast Reconstr Surg. 2001;107(1):9-14.

14. Cable BB, Canady JW, Karnell MP, Karnell LH, Malick DN. Pharyngeal flap sugery: long-term outcomes at the University of Iowa. Plast Reconstr Surg. 2004;113(2):475-8.

15. Liedman-Boshko J, Lohmander A, Persson C, Lith A, Elander A. Perceptual analysis of speech and the activity in the lateral pharyngeal walls before and after velopharyngeal flap surgery. Scand J Plast Reconstr Surg Hand Surg. 2005;39(1):22-32.

16. Dailey SA, Karnell MP, Karnell LH, Canady JW. Comparison of resonance outcomes after pharyngeal flap and furlow double-opposing z-plasty for surgical management of velopharyngeal incompetence. Cleft Palate Craniofac J. 2006;43(1):38-43.

17. de Buys Roessingh AS, Cherpillod J, Trichet-Zbinden C, Hohfeld J. Speech outcome after cranial-based pharyngeal flap in children born with total cleft, cleft palate, or primary velopharyngeal insufficiency. J Oral Maxillofac Surg. 2006;64(12):1736-42. 
18. Van Lierde KM, De Bodt M, Van Borsel J, Wuyts FL, Van Cauwenberge P. Effect of cleft type on overall speech intelligibility and resonance. Folia Phoniatr Logop. 2002;54(3):158-68.

19. Wells MD, Vu TA, Luce EA. Incidence and sequelae of nocturnal respiratory obstruction following posterior pharyngeal flap operation. Ann Plast Surg. 1999;43(3):252-7.

20. Liao YF, Chuang ML, Chen PK, Chen NH, Yun C, Huang CS. Incidence and severity of obstructive sleep apnea following pharyngeal flap surgery in patients with cleft palate. Cleft Palate Craniofac J. 2002;39(3):312-6.

21. Yamashita RP, Trindade IEK. Long-term effects of pharyngeal flaps on the upper airways of subjects with velopharyngeal insufficiency. Cleft Palate Craniofac J. 2008;45(4):364-70.

22. Trindade IEK, Fukushiro AP, Yamashita RP, Sampaio ACM, Trindade Junior AS. Prevalence of sleep-disordered breathing in middle-aged cleft adults with pharyngeal flaps. X International Congress on Cleft Palate and Related Craniofacial Anomalies; 2005 Sep 4-8; Durban. Proceedings. Durban: International Confederation for Cleft Palate and Related Craniofacial Anomalies; 2005.

23. Warren DW. Aerodynamics assessment and procedures to determine extent of velopharyngeal inadequacy. In: Bzoch KR. Communicative disorders related to cleft lip and palate. 4a ed. Austin: Pro-ed; 1997. p. 411-37.
24. Hosmer DR, Lemeshow S. Applied Logistic Regression. New York: John Wiley \& Sons; 1989.

25. Shprintzen RJ, Lewin ML, Croft CB, Daniller AI, Argamaso RV, Ship AG, et al. A comprehensive study of pharyngeal flap surgery: tailor made flaps. Cleft Palate J. 1979;16(1):46-55.

26. Argamaso RV, Shprintzen RJ, Strauch B, Lewin ML, Daniller AI, Ship $\mathrm{AG}$, et al. The role of lateral pharyngeal wall movement in pharyngeal flap surgery. Plast Reconstr Surg. 1980;66(2):214-9.

27. Friedman HI, Haines PC, Coston GN, Lett ED, Edgerton MT. Augmentation of the failed pharyngeal flap. Plast Reconstr Surg. 1992;90(2):314-8

28. Morris HL, Bardach J, Jones D, Christiansen JL, Gray SD. Clinical results of pharyngeal flap surgery: the Iowa experience. Plast Reconstr Surg. 1995;95(4):652-62.

29. Hall CD, Golding-Kushner KJ, Argamaso RV, Strauch B. Pharyngeal flap surgery in adults. Cleft Palate Craniofac J. 1991;28(2):179-82.

30. Sampaio ACM, Trindade IEK, Genaro KF, Yamashita RP, Trindade Junior AS. Dimensões das vias aéreas na respiração de repouso: área nasal e faríngea. I Congresso Científico da USP; 1997 Nov 28-29; Bauru. Livro de Resumos. Bauru: USP; 1997. 